\title{
Semiconductor Quantum Dots as In-Vivo Imaging Agent
}

\section{Joydeep Dhar*}

Department of Chemistry, Birla Institute of Technology Mesra, Jharkhand, India

Received: 烈June 26, 2018; Published: 眥 July 02, 2018

*Corresponding author: Joydeep Dhar, Department of Chemistry, Birla Institute of Technology Mesra, Ranchi-835215, Jharkhand, India

\section{Short Communication}

Inability to early diagnosis is a major concern for the treatment of fatal disease like cancer. Early diagnosis and treatment enhances the scope of disease curability. The accurate identification, realtime monitoring and targeting the cancerous tissues in a precise manner hold the key for longer progression free survival of a patient. Among different diagnostic techniques, fluorescence based minimally invasive bio-imaging techniques are considered to be ideal to have clear understanding about the physiological processes of the infected tissues as well as to reduce physical and mental stress of a patient. Super-resolution fluorescence microscopy has improved the spatial optical resolution of biological molecules, living cells and tissues with the use of highly fluorescent inorganic semiconductor nanocrystals, also known as quantum dots (QDs), with sizes ranging from $2 \mathrm{~nm}$ to $15 \mathrm{~nm}$ [1]. These nanocrystals comprised of elements belong to groups II-VI (eg, CdSe and CdTe), groups III-V (eg, InP), groups IV-VI (eg, PbS and PbSe) [2]. The QDs have several unique optical properties which make them far superior than organic chromophores as fluorescent probes in fluorescence microscopy. The exciting optical properties of QDs exhibiting due to their size which is smaller than their exciton Bohr radius [3]. For example, QDs have high molar extinction coefficients, high quantum efficiency ( $>50 \%$ ), narrow emission spectra, higher excited state lifetimes and high resistance to photo-bleaching [4]. In addition to this, QDs are at least 15 times brighter than organic dyes under the same experimental conditions [5]. Such unique optical properties are utilized to enhance the signal-to-background ratio for microscopic imaging [6]. Moreover, the optical band gap and in turn, emission wavelength of QDs can be systematically tuned from the visible to near-infrared (NIR) spectral region by simply manipulating their size, shape, composition, and structure as shown in Figure 1 [7]. Thus, optical tunability of QDs renders opportunity in multiplexed and real-time imaging [8]. Due to large absorption cross section, QDs are also potential candidates for twophoton imaging as compared to some organic dyes [9].

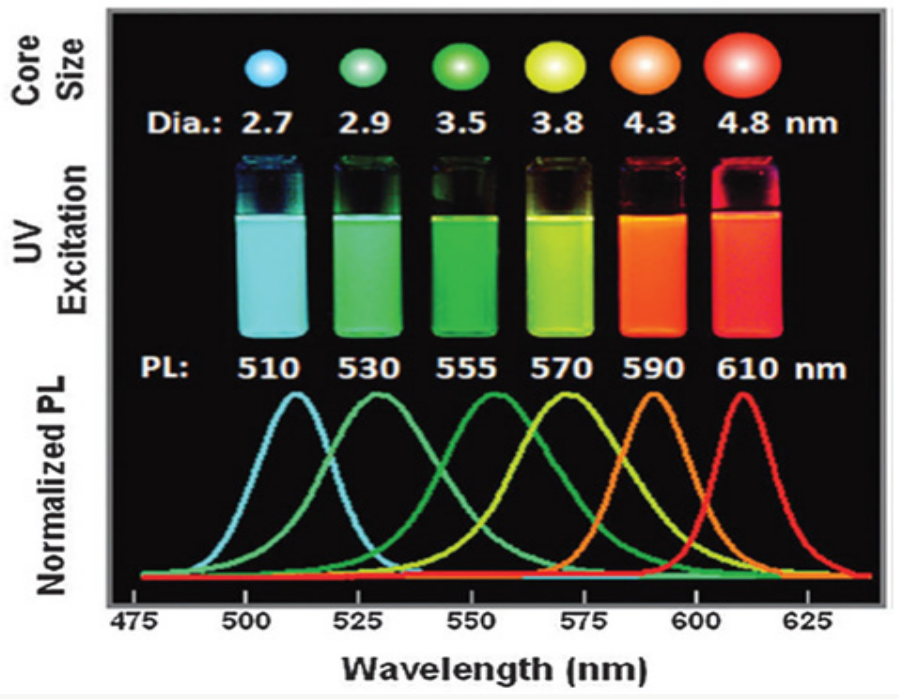

Figure 1: Size dependent emission properties of core-shell CdSe@ZnS QDs (Adapted from Reference 7).

Highly fluorescent QDs are used to monitor the dynamic changes of a tumor microenvironment contributing towards the research of cancer invasion mechanism and guiding for better clinical therapy. Therefore, as compared to other conventional imaging 
techniques, a molecular QD based targeted therapy offers various advantages. First, multitude of imaging labels or combinations of labels can be attached to a single nanostructure, which can lead to dramatic signal amplification. Second, multiple, potentially different, targeting ligands on the QD can provide enhanced binding affinity and specificity. Third, it provides opportunity to integrate a specific biomarker to bypass biological barriers to enhance targeting efficacy. Finally, the attachment of different targeting ligands, therapeutic drugs, and other agents allow the effective and controlled delivery of therapeutics in patients, which can be noninvasively monitored in real time. Biofunctionality of QDs is achieved in presence of the water soluble ligand shell making QD hydrophilic and stable in a range of biological media including fluids, tissues and within the cells. Stabilizing ligand shell is important to maintain the optical properties of QD which is strongly affected by the local environment of the biological media. These optical properties include onset of absorption, emission wavelength, and photo luminescence quantum yield (PLQY) and fluorescence lifetime. Nowadays, QDs provide the best compromise between size and photo physical properties for cell analysis and imaging applications. Under in vivo conditions, QD probes can be delivered to the tumors by both passive and active targeting mechanisms. In the passive targeting mode, QDs accumulate preferentially at tumor sites through an enhanced permeability and retention (EPR) effect. This effect is believed to arise due to two factors:
I. Angiogenic tumors produce leaky vascular endothelial growth and

II. Tumors tissue lacks an effective lymphatic drainage system, allowing QD to accumulate and penetrate into the affected tissues. For active tumor targeting, the antibodyconjugated QDs are used to target a specific membrane antigen over-expressed at the tumor site.

Figure 2 shows the real-time imaging and tracking of antibodyconjugated QDs towards prostate-specific membrane antigen (PSMA) on the surface of living cells with improved sensitivity and resolution [10]. Fluorescence signals emanating from QDs clearly indicate the growth of prostate tumor in a live mouse. In recent years, several studies have demonstrated the utility of surface functionalized QDs as biomarker in detection of tumor growth through in vivo imaging [7,11]. In spite of QDs exhibiting excellent fluorescence intensity and photo stability the biocompatibility and cytotoxicity are the two most important concerns associated with the use of QD as biomarkers for in vivo imaging. Over the years, the cytotoxicity data analysis has provided a large diversity of data set based on different biocompatibility experimental design studies using a wide array of QDs. The one to one correlation is still lacking and needs more rigorous systematic analysis to reach a logical conclusion on a hugely potential field of nanobioscience.
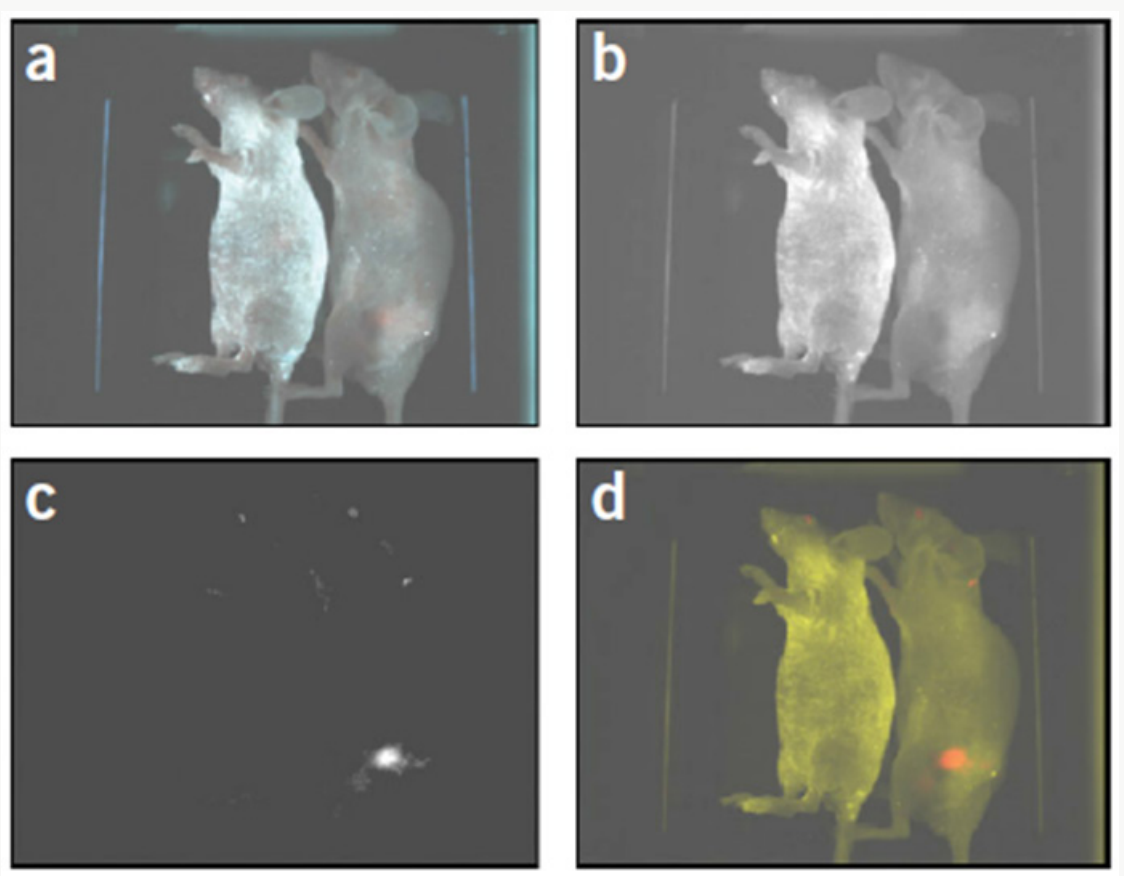

Figure 2: In vivo spectral imaging of QD-PSMA Ab conjugates in a mouse harboring C4-2 tumor xenografts. Presence of orangered fluorescence signals indicates prostate tumor growing in a live mouse. Control studies with a healthy mouse (no tumor) and the same amount of QD injection showed no localized fluorescence signals (left). (a) Original image; (b) Unmixed auto fluorescence image; (c) Unmixed QD image; and (d) Super-imposed image. (Adapted from Reference 10).

\section{References}

1. Bruchez M, Moronne M, Gin P, Weiss S, Alivisatos AP (1998) Semiconductor nanocrystals as fluorescent biological labels. Science 281(5385): 2013-2016.
2. Farkhani SM, Valizadeh A (2014) In IET Nanobiotechnology Institution of Engineering and Technology Vol. 8.

3. Chan WCW, Maxwell DJ, Gao X, Bailey RE, Han M, et al. (2002) Luminescent quantum dots for multiplexed biological detection and imaging. Curr Opin Biotechnol 13(1): 40-46. 
4. Chan WCW, Nie S (1998) Quantum dot bioconjugates for ultrasensitive nonisotopic detection. Science 281(5385): 2016-2018.

5. Altinoglu EI, Adair JH, (2010) Wiley Interdisciplinary Reviews: Nanomedicine and Nanobiotechnology 2(5): 461.

6. Dubertret B, Skourides P, Norris DJ, Noireaux V, Brivanlou AH, et al. (2002) In vivo imaging of quantum dots encapsulated in phospholipid micelles. Science 298(5599): 1759-1762.

7. Wegner KD, Hildebrandt N (2015) Quantum dots: bright and versatile in vitro and in vivo fluorescence imaging biosensors. Chem Soc Rev 44(14): $4792-4834$

Submission Link:

DOI: $10.32474 /$ DDIPIJ.2018.01.000124

\section{This work is licensed under Creative Commons Attribution 4.0 License \\ (c) (1)}

Submit Article
8. Sharma P, Brown S, Walter G, Santra S, Moudgil B (2006) Nanoparticles for bioimaging. Adv Colloid Interface Sci 123-126: 471-85.

9. Michalet X, Pinaud FF, Bentolila LA, Tsay JM, Doose S, et al. (2005) Quantum dots for live cells, in vivo imaging, and diagnostics. Science 307(5709): 538-544.

10. Gao X, Cui Y, Levenson RM, Chung LWK, Nie S (2004) In vivo cancer targeting and imaging with semiconductor quantum dots. Nat Biotechnol 22(8): 969-976.

11. Zrazhevskiy P, Sena M, Gao X (2010) Designing multifunctional quantum dots for bioimaging, detection, and drug delivery. Chem Soc Rev 39(11): 4326-4354.

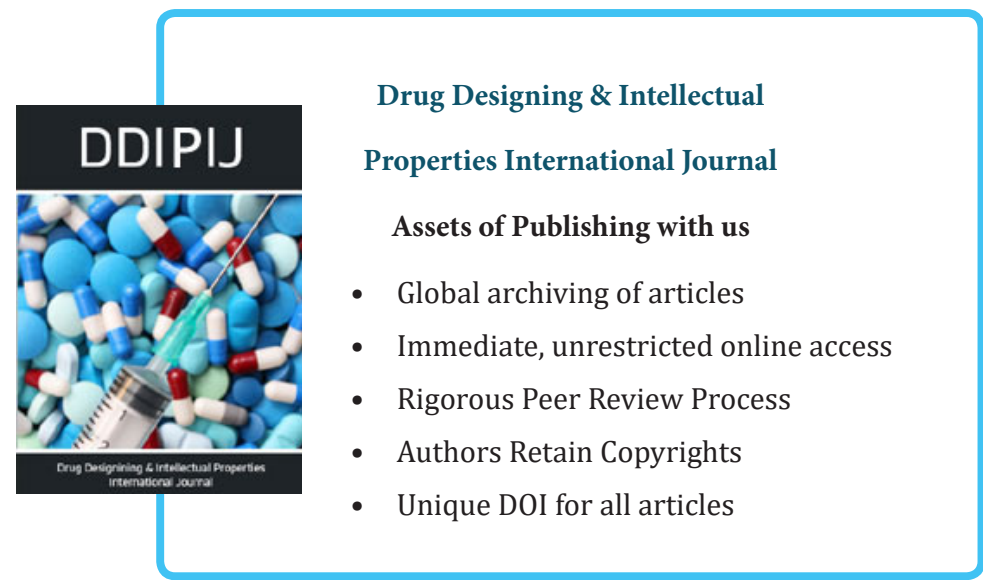

